\title{
Extending the Selective MPEG Encryption Algorithm PVEA
}

\author{
Li Weng, Karel Wouters and Bart Preneel \\ Department of Electrical Engineering \\ IBBT-IPEA*, Katholieke Universiteit Leuven \\ Kasteelpark Arenberg 10, 3001 Leuven-Heverlee, Belgium \\ Email: $\{$ li.weng, karel.wouters, bart.preneel\}@esat.kuleuven.be
}

\begin{abstract}
Currently, the requirements on MPEG encryption algorithms include: fast speed, format compliance, a wide range of security levels, easy implementation, etc. However, promising candidates that possess all these properties have not appeared. In this work we design a selective encryption algorithm for MPEG video with the aim to address all these problems.
\end{abstract}

\section{Introduction}

Multimedia data is being distributed via digital links with a sharply growing trend. This motivates the emerging need for corresponding confidentiality. Due to the bulky size, applying standard ciphers to multimedia data tends to be inefficient for real-time, low-power or high-definition scenarios. Therefore, selective encryption initially appeared as means to relieve computational cost. After the emphasis by earlier work, some principles have become well-known and widely adopted: (1) in predictive coding, intra-coded content is more essential than predictive-coded one; (2) in scalable coding, base layers are more essential than enhancement ones; (3) in progressive coding, significant bit-planes are more essential than less significant ones. Accordingly, encryption can be selectively applied to these partitions and result in less computation. In this work, we do not reemphasize the above knowledge ( see e.g. [3] for a review); instead, we design a selective encryption algorithm for MPEG video with the following goals:

- Compliant to MPEG format;

- Significantly faster than standard cipher encryption;

${ }^{*}$ The IBBT - IPEA project is co-funded by the IBBT (Interdisciplinary Institute for BroadBand Technology), a research institute founded by the Flemish Government in 2004, and the involved companies and institutions (Philips, IPGlobalnet, Vitalsys, Landsbond onafhankelijke ziekenfondsen, UZ-Gent). https://ipea.ibbt.be
- No influence on compression ratio or quality;

- A wide range of adjustable security levels.

These properties are attracting more attention in the design of selective encryption algorithms $[1,7,3]$. New designs more or less take some of them into consideration. However, to the best of our knowledge, an algorithm that fulfills all of them, has not appeared. Our design might serve as a promising template solution in the presence of a "chaotic" video encryption domain.

\section{The new design}

This design is an extension to the perceptual video encryption algorithm (PVEA) [1]. PVEA is a fast selective encryption algorithm for MPEG video. It has many interesting features, such as full format compliance and no influence on compression; it offers many security (perceptibility) levels. These features are achieved by applying standard cipher encryption to the following elements in the MPEG bitstream:

- Intra DC coefficients - corresponding to rough view of the video;

- Sign bits of non-intra DC and AC coefficients, and ESCAPE DCT coefficients - corresponding to a small portion of details of the video;

- Sign bits and residues of motion vectors - corresponding to visual quality related to motions.

The above elements are encoded by fixed length codes, so encrypting them does not affect the format and the size; each of them has different perceptual importance, so multiple levels of security can be achieved by encrypting any of them in a freely combined way. Further trade-offs between computational cost and security can be achieved by running the encryption in partial time and/or to partial data (e.g., Iframes, significant bits). These parts occupy a small portion of the whole stream, so the computational cost is generally 
low. Compared to other approaches, since a standard cipher is used, PVEA has the highest possible security for protecting corresponding elements.

However, PVEA was designed as a perceptual encryption scheme. Due to limited perceptual importance of encrypted elements, it only degrades visual quality to some extent, which is not sufficient for protecting highly confidential content. It is vulnerable to error concealment attack (ECA): e.g., by setting intra DC coefficients to zero and all the sign bits of $\mathrm{AC}$ coefficients to one, object contours sometimes remain and are intelligible (Fig. 1c).

Our new design adopts PVEA as infrastructure due to its good properties. Based on that, we encrypt one more element to further diffuse the contour information:

- AC coefficients (excluding sign bits) - corresponding to most details of the video.

This is a quite natural choice, since the four candidate elements together compose a partition of all the visual information in the bitstream. By encrypting all or some of them, we not only cover a higher security level, but also get more intermediate levels.

During compression, the AC coefficients are first encoded by a run-length code; run-level pairs are then represented by a variable length code (VLC) in the final bitstream. To maintain both the format and the size, we encrypt AC coefficients by spatially shuffling VLC codewords after run-length coding. Since it is just a permutation, it can be easily implemented. In the following, we discuss the deployment in detail with respect mainly to security and speed.

\section{Security and speed co-design}

\subsection{On brute-force attack}

This VLC codeword shuffling scheme requires a permutation $\left\{P_{1}, P_{2}, \ldots, P_{k}\right\}$, where $P_{i}$ is a position index and $k$ is the number of indices. It can be obtained by modifying the output of a stream cipher. We define $k$ as the size of the permutation. It is well-known that there are $k$ ! different permutations. To resist brute-force attack, $k$ ! should be sufficiently large. However, we cannot set $k$ arbitarily large, because the delay caused by de-permutation and the buffer needed are proportional to $k$. For real-time or memory-constrained applications, this might be a concern. For example, we may choose $k=64$, so the size of search space for brute-force attack is $64 !>2^{295}$, which is sufficient in many scenarios. On the other hand, if $k$ is too small, we have threat not only from brute-force attack, but also from frequency-based attack [4]. Therefore, generally $k$ should span more than one $8 \times 8$ block. Note that for the de-permutation to work, the borders (e.g., End-of-Block symbol in MPEG-2) between blocks should be kept ${ }^{1}$.

\subsection{On plaintext attack}

Since a permutation only changes the positions of input values, if all these values are distinct, the permutation is certainly known by comparing ciphertext and plaintext. In this sense, any permutation scheme is vulnerable to chosen plaintext attack. However, such attack can be avoided by a good protocol and is relatively rare for multimedia applications.

To resist known plaintext attack, it is not enough to use only one permutation. As indicated by an equation in [2], if we have $n$ ciphertext-plaintext pairs for the same permutation of size $k$, then for each index in the permutation, on average we get $E=1+(k-1) / L^{n}$ estimations (including the true position), where $L$ is the number of different values each position can take (assuming independent uniform distribution). When $E$ is less than 1.5, the estimation result is generally acceptable. As seen before, it is sufficient to set $k$ no more than 64 to resist brute-force attack. However, in e.g. MPEG-2, there are more than a hundred VLC codewords; although they are not uniformly distributed, if we assume $L>50$, we may still get a good approximation of the permutation even when $n=2$ ! The equation also reveals that it is less secure to apply permutation alone to higher level visual elements, such as $8 \times 8$ blocks or $16 \times 16$ macroblocks, because in that case, $L$, the number of different contents for a (macro)block, is too large.

Consequently, the permutation should frequently change. We can generate a new permutation by running the stream cipher. Assuming $k=64$, the length of the permutation is $k \log _{2} k=384$ bits. If a stream cipher outputs one byte at a time, we have to run it 48 times, which can be slow. Besides speed, security is still an issue. For the adversary there is always the chance to know some (or even all) permutations if enough ciphertext-plaintext pairs are collected. In particular, we can expect two attacks: 1) the adversary knows the previous permutation and wants to guess the current one; 2) the adversary knows all permutations and wants to guess the current one. We denote the complexity for the first attack as $N_{p 1}$; the complexity for the second attack as $N_{p 2}$, which is actually the total number of permutations in use. These two parameters can benchmark the security of a permutation updating procedure. For the above updating scheme, we get the highest security, i.e., $N_{p 1}=N_{p 2}=k$ !, at the cost of a low speed. If we neglect the time for conducting the permutation and assume the average length of a VLC codeword is $x$ bits, then this scheme protects $k x$ bits by

\footnotetext{
${ }^{1}$ A block may contain more than 64 coefficients after permutation, but this is no problem for a decoder with least error resilience.
} 
encrypting $k \log _{2} k$ bits. Therefore we only gain speed if $x \gg \log _{2} k$, which is not always true. In the following, we explore ways to balance speed and security in permutation updating.

If the highest security is not affordable due to the low speed, we may run a cipher less often. One solution is given in [8], where a master bit sequence is generated by a stream cipher at the beginning, corresponding to a permutation of size $k$; later, for every group of $k$ VLC codewords, their sign bits are encrypted first by a standard cipher, then the resulted ciphertext is encrypted again by DES in electronic codebook (ECB) mode. The new ciphertext is replicated and concatenated to get a bit sequence with the same length as the master sequence, then they are XORed (and slightly adjusted) to get the current permutation, which is used to permute those VLC codewords. This scheme protects $k$ VLC codewords by encrypting $k$ bits. Assuming a VLC codeword has 6 bits, the saving in computation is about $5 / 6$ compared to encrypting all VLC codewords by DES. As a trade-off, the security is slightly lowered: $N_{p 1}=N_{p 2}=2^{k}$. An advantage of this scheme is selfsynchronization, i.e., current decryption only depends on current data. However, such a scheme is not so flexible because in ECB mode $k$ has to be a multiple of 64 . Since $k$ is also a benchmark of delay, we only get a few choices of bundled security-delay combinations. It might be useful to set $k$ freely.

We extend this scheme by using the output feedback (OFB) mode. We encrypt $k^{\prime}$ bits and permute $k$ VLC codewords, and keep the rest procedure the same. As a result, $N_{p 1}=N_{p 2}=2^{k^{\prime}}$ and $k$ can be separately chosen and $k / k^{\prime}$ does not have to be a multiple of 64 . When $k^{\prime}>k$, we can take extra bits from the ciphertext of PVEA. In OFB mode, even $k^{\prime}$ is less than 64, DES still encrypts 64 bits every time. If we only use the first $k^{\prime}$ bits of ciphertext, some computation is wasted, so we may store the whole 64 bit ciphertext and use it later. To deal with loss or error, we put the synchronization at higher levels and propose to use a hybrid counter and OFB (CT-OFB) mode for e.g. each frame. That means, at the beginning of a frame, a counter is used as the initial vector, then the cipher is run in OFB mode. For the next frame, the counter is incremented and followed by OFB mode again. This mode preserves the random frame access ability of the underlying standard. We can jump to other frames according to the correspondence between the counter and the frame number. Note that the ciphertext in use for permutation updating should also be restricted within e.g. each frame.

Based on the above scheme, we can still improve the speed from two aspects. First, we see that DES is used to update the permutation. Since DES always needs input, which is the ciphertext of PVEA, time has to be spent on collecting and managing the input. This task can be avoided if we replace the block cipher with a stream cipher. We can use the stream cipher as a secure pseudo-random number generator to produce random bits and update the permutation in the same way. Another advantage of a stream cipher is that it is normally faster than a block cipher.

Second, note that we do not really get a permutation if we XOR random bits with the master sequence. The result has to be adjusted according to some pre-determined rules to become a permutation. This procedure is inefficient and also can be avoided. A natural way to quickly generate different permutations is to use the swap operation. If a permutation is updated by swapping its elements, the result is also a permutation, thus no extra adjustment is needed. In this way the speed can be further increased. A swap can be defined as a pair of different integer indices $\{m, n\}, m \neq n$, which correspond to the positions of the to-be-swapped elements in a sequence. For each round of updating, we generate $x$ swaps by a stream cipher and apply them to the previous permutation. Therefore we only have to do the adjustment once to get the initial permutation.

By using swapping, any possible permutation can be generated, thus $N_{p 2}$ becomes very large, ideally $k$ !. However, if we do not apply enough swaps in each round of updating, $N_{p 1}$ tends to be small. For example, if we only use one swap, then $N_{p 1}=\left.\left(\begin{array}{c}k \\ 2\end{array}\right)^{x}\right|_{x=1}$, which is usually small. Therefore we have to use several swaps to get a reasonably big $N_{p 1}$. However, there is again a speed problem. Assume we use RC4 and run it twice to get a swap, with each index ranging from 0 to 255 . According to the data in [5, pp. 195], the speed of RC4 is 4 times faster than DES. That means to maintain a significant speed advantage over DES, we should not run RC4 more than 4 times for each round, which only corresponds to 2 swaps. Assuming $k=64$, then $N_{p 1}=\left(\begin{array}{c}64 \\ 2\end{array}\right)^{2}<2^{22}$, which might not be sufficient. To increase the security but maintain the speed, we use the following alternative scheme.

- First round: run RC4 to get $x$ swaps; store last $x-1$ of them in a buffer after use;

- A later round: get $x-1$ swaps by incrementing elements of previous $x-1$ swaps in the buffer; run RC4 to get another swap and put it in the buffer after use.

In this way, we run RC4 less often but still get enough swaps. The purpose of incrementation is to avoid that the same swap is consecutively applied. Assuming the time for the incrementation is negligible, the speed improvement over DES is more than $50 \%$. The price to pay for this scheme is that once it is broken in a round, $N_{p 1}$ drops from $\left(\begin{array}{l}k \\ 2\end{array}\right)^{x}$ to $\left(\begin{array}{l}k \\ 2\end{array}\right)$ in following rounds, which might be an acceptable compromise. 


\section{Security evaluation and implementation}

To evaluate the security enhancement, we apply the permutation scheme to a $512 \times 512$ gray-scale Lena picture ${ }^{2}$ and use a visual quality assessment metric - the structural similarity (SSIM) [6] ${ }^{3}$ to evaluate the intelligibility of the encrypted picture. This metric takes the original and the encrypted pictures as input, and outputs a fraction between zero (totally different) and one (identical). We choose this metric because structures usually give most information in a picture. First we simulate PVEA by setting DC coefficients and sign bits of AC coefficients to random values. We get an SSIM score of 0.34 (Fig. 1a); further, we randomly permute every $k=36$ run-level pairs and get a similar score (Fig. $1 \mathrm{~b})$, which decreases slowly with $k$. That means PVEA has already destroyed most structures. More interestingly, after ECA is applied to Fig. 1a, the score has risen to 0.42 (Fig. 1c); while if applied to Fig. 1b, the score remains (Fig. 1d) and decreases with $k$. Although the new scheme enhances security slowly, it helps to resist ECA. In general, the security enhancement by permutation depends on visual content (e.g. the size), and also on (de)compression, so there might not be definite measurement.

To impelement the whole encryption scheme, we need a cipher for PVEA, a stream cipher for generating the initial permutation and a cipher for updating the permutation, each requiring a key and its own synchronization scheme. Apparantly, it is most convenient to use the same stream cipher for all tasks. In that case, only one key is needed. The synchronization also becomes simpler. For example, we can reset the stream cipher at the beginning of a frame with the concatenation of the secret key and the frame number. Additionally, note that a permutation might not be good if it only changes a few positions, so we may need to check the "quality" of a permutation after it is generated.

\section{Conclusions}

We design a selective MPEG encryption scheme by combining PVEA and VLC codeword permutation. It can be summarized as protecting fixed length codes by standard cipher encryption and variable length codes by permutation. The idea is quite general, thus can be applied to various MPEG-like video standards. It is fast, format compliant and has adequate security levels. We pay particular attention to resist known plaintext attack and propose to update the permutation by a cipher: we extend the approach in [8] by using a hybrid CT-OFB mode, offering more flexible security/speed trade-offs; we further improve the speed by using a stream cipher and the swap operation. Our simulation

\footnotetext{
${ }^{2}$ After DCT, it is quantized by a JPEG quantization matrix, Zigzag scanned, and represented as run-level pairs to simulate intra compression. After encryption, each block takes first 64 DCT coefficients for decoding.
}

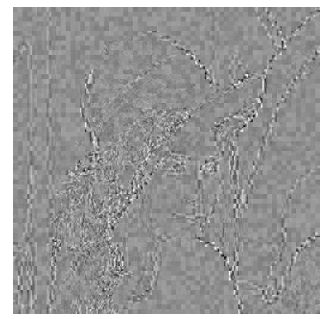

(a) PVEA

SSIM $=0.34$

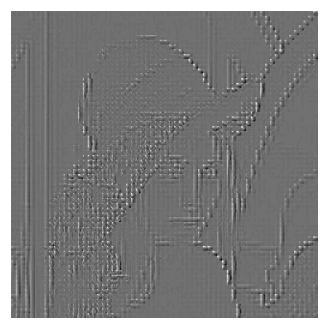

(c) PVEA+ECA

SSIM $=0.42$

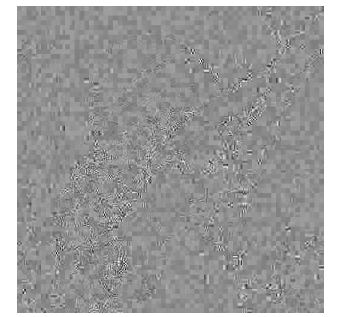

(b) new scheme

SSIM $=0.34$

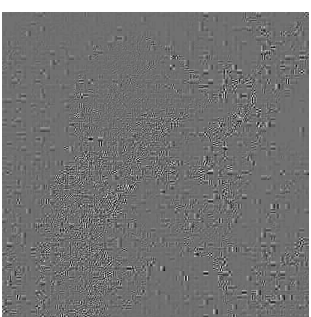

(d) new scheme+ECA

SSIM $=0.346$

\section{Figure 1. Error concealment attack (ECA)}

shows the new scheme can indeed achieve higher security than PVEA. In the future we will implement this scheme and give speed evaluation.

\section{References}

[1] S. Li, G. Chen, A. Cheung, and B. Bhargava. On the design of perceptual MPEG-video encryption algorithms. ArXiv Computer Science e-prints, 2005.

[2] S. Li, C. Li, G. Chen, D. Zhang, and N. Bourbakis. A general cryptanalysis of permutation-only multimedia encryption algorithms. Cryptology ePrint Archive: Report, 2004.

[3] X. Liu and A. M. Eskicioglu. Selective encryption of multimedia content in distribution networks: challenges and new directions. In Proc. Communications, Internet, and Information Technology, 2003.

[4] L. Qiao and K. Nahrstedt. Comparison of MPEG encryption algorithms. Computers and Graphics, 22(4):437-448, 1998.

[5] W. Stallings. Cryptography and Network Security: Principles and Practices. Upper Saddle River, NJ: Prentice Hall, 3rd edition, 2003.

[6] Z. Wang, A. Bovik, H. Sheikh, and E. Simoncelli. Image quality assessment: From error visibility to structural similarity. IEEE Trans. Image Processing, 2004.

[7] J. Wen, M. Severa, W. Zeng, M. Luttrell, and W. Jin. A format compliant configurable encryption framework for access control of multimedia. In Proc. IEEE Workshop on Multimedia Signal Processing, 2001.

[8] W. Zeng, J. Wen, and M. Severa. Fast self-synchronous content scrambling by spatially shuffling codewords of compressed bitstreams. In Proc. IEEE Inter. Conf. Image Proc., 2002.

\footnotetext{
${ }^{3}$ Source code at: http://www.cns.nyu.edu/ lcv/ssim/
} 